\title{
La matemática escolar y los estadios de desarrollo *
}

\section{Kevin F. Collis}

Traducción: Pablo del Rio

En la aplicación de la teoría de Piaget sobre el desarrollo intelectual del niño a la escuela, se ha trabajado más y son mucho más conocidos los dos puntos extremos de la escala: la etapa preescolar (preoperatoria) y el B.U.P. (pensamiento formal), quizá a causa de que las fronteras de la programación educativa suelen ser más accesibles a un trabajo psicopedagógico hecho desde fuera.

Este artículo viene, sistemáticamente, a afrontar para el ciclo completo y con especial énfasis en las etapas de E.G.B., la aplicabilidad de Piaget a las matemáticas escolares, a partir de abundantes y serios trabajos.en el contexto escolar.

- «School Mathematics and Stages of Development». En Modgil, S. y Modgil, C. (edś.) Towards a Theory of Psychological Development. Windsor, N. J.: N.F.E.R. Publishing Co. 1980, pp. 635.67 1. Reproducido con autorización. (C) de esta traducción, Infancia y Aprendizaje, 1982. 


\section{LA MATEMATICA ESCOLAR: LA TAREA}

Podemos considerar las matemáticas escolares como un sistema o estructura lógica de relaciones cuya base formada por un conjunto definido de elementos y un método claramente definido para operar con ellos. La necesidad de comunicar parte de la estructura o del sistema a los demás, da origen a un simbolismo formal que incluye tanto a los elementos como a las operaciones. Podemos servirnos del enunciado matemático $2(x+y)=2 x+2 y$ para ilustrarlo. Los elementos implicados en el enunciado son números y variables y están claramente definidas las operaciones a efectuar con los elementos: multiplicación y adición. Los símbolos $2, \mathrm{x}$, $\mathrm{y}$, son abstracciones que nos permiten un modo abreviado de comunicar nuestro pensamiento a los demás y, por último, el propio enunciado indica la existencia de una conexión entre las dos partes de la estructura, aquella relativa a la suma y aquella otra relativa a la multiplicación.

Y ahí precisamente está la dificultad. Esta descripción que acabamos de hacer es la imagen que ven el matemático o el maestro y que confían hacer ver en último término a sus alumnos. La tarea que el niño ha de hacer para desarrollar su sofisticación matemática hasta este punto ha de ser enorme, si tenemos en cuenta cual es su punto de partida. Tomemos por ejemplo el niño que comienza el primer ciclo de la escuela primaria y que cumple el criterio básico de encontrarse al principio del nivel de desarrollo de las operaciones concretas y veamos qué cosas ha de establecer para iniciar su inducción en el campo de las matemáticas.

Debemos dejar claro en primer lugar que el sistema formal de las matemáticas no existe para el niño: existe la experiencia y el pensamiento opera en los términos de esta experiencia. Si estamos de acuerdo en ésto, de ahí se sigue que todos los componentes de las matemáticas que hemos descrito antes, deben considerarse en principio, como una mera representación de lo que puede observarse clara y definidamente en la realidad concreta. Consideremos un aspecto, por ejemplo la idea básica de cardinalidad de un conjunto y la operación elemental de adición utilizando números de cuenta.

Hacia los 6 ó 7 años, los niños habrán tenido ya sin duda muchas experiencias con agrupamientos de elementos en su entorno, por ejemplo, el grupo familiar, niños/niñas, perros/gatos, etc. Y distinguen estos grupos por ciertos atributos que han aprendido a reconocer y que generalmente tienen algún interés intrínseco para ellos. Parecería razonable comenzar a desarrollar entonces la idea matemática de conjunto, especialmente en la medida en que este concepto puede utilizarse para unificar la estructura global de las matemáticas tal como habitualmente se enseñan en la escuela primaria. Hacer esto requiere, sin embargo, que los niños 
concentren su atención en los atributos del conjunto que están re. lacionados con la medición de su cardinalidad. En sí misma, quizá no sea ésta una tarea sencilla, puesto que la mayoría de los conjun- tos de objetos poseen atributos mucho más interesantes para el niño pequeño que su número cardinal. Tras lograr ese reenfoque, el niño podrá darse cuenta de que ese atributo es el único común a todos los conjuntos y, aún más, iel único que parece interesar al maestro!

La siguiente tarea es la de medir y registrar con fiabilidad el número cardinal de un conjunto. El medio obvio para lograr el primero de estos objetivos es contar, y el de alcanzar el segundo, los números escritos. Contar exige el recitado de números en una secuencia específica, manteniendo sucesivamente una correspondencia uno-a-uno entre los números recitados y los elementos del conjunto. Una vez dominada esta técnica, el sujeto necesita un medio para registrar el resultado, un símbolo único que represente únicamente el número contado. Comienza así la simbolización implícita en las matemáticas, el número, por ejemplo 4, que nos recuerda un conjunto estándar de elementos a los que puede ponerse en correspondencia uno-a-uno con cualquier otro conjunto que tenga una medida de 4 en números cardinales. Podrá considerarse que la próxima tarea en la construcción de estructura sería la de encontrar un modo de manejar el número de elementos existentes cuando se unen dos conjuntos disjuntos de elementos. Esto implica el unir, bien física, bien mentalmente, los conjuntos en cuestión considerando el resultado de la unión como un solo conjunto. Es obvio que el número cardinal podrá hallarse contando los elementos que intervienen en la unión, pero contamos con la alternativa de mantener ante nosotros los conjuntos originales y considerar simplemente los números en cada uno de los conjuntos. Por ejemplo, si tenemos que unir los conjuntos de cruces A y B que aparecen en el diagrama, podríamos contar todas las cruces y convenir en que hay cinco elementos en la unión. Por otra parte; podríamos

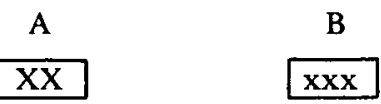

decir, si quisiéramos, que hay dos elementos en A y tres elementos en $\mathrm{B}$ y hallar un símbolo que represente a ambos números juntos, un análogo de lo que sería unir los elementos de los conjuntos. En ese caso el resultado estaría representador por $2+3$. Definiríamos así la operación de adición como $n(A)+n(B)=(A \cup B)$ donde $n(A)$ significa el número cardinal del conjunto $\mathrm{A}$ etc., $\mathrm{y}$ donde $\mathrm{A}$ y $\mathrm{B}$ son conjuntos disjuntos.

Se comprobará que extender esta línea de pensamiento nos permitiría producir proposiciones matemáticas equivalentes. Por ejemplo, podríamos considerar que los siguientes elementos 
$\widehat{\mathrm{XX}} \widehat{\mathrm{XX}}$ pertenecen a conjuntos disjuntos en una diversidad de maneras, dos de las cuales están representadas por los trazos. Si consideramos los trazos superiores, $2+.2$ sería una manera acertada y apropiada de expresar la cardinalidad de esta unión; mientras que si consideramos los trazos inferiores, también resulta apropiada la expresión $3+1$. Son ambos dos medidas acertadas de la cardinalidad presente y por tanto es razonable hacer la siguiente afirmación matemática $2+2=3+1$.

Lo que hemos pretendido establecer hasta aquí es que en todas las etapas del aprendizaje por el niño de las matemáticas escolares, la naturaleza de la materia de contenido, asegura que el niño se vea envuelto en una lucha con un sistema lógico basado en un conjunto de elementos y con un método de operar con ellos, así como de comunicar después a los demás los resultados. Si deseamos elaborar una estructura de comprensión de las matemáticas es preciso trabajar en este marco. Pero el problema, por supuesto, es que los distintos niveles del funcionamiento cognitivo ponen límites a la sofisticación que pueda esperarse en distintos estadios del desarrollo. Proponemos ahora volver a ocuparnos de los niveles de funcionamiento cognitivo en general y volver otra vez sobre ellos, ya en el contexto especial del aprendizaje de las matemáticas.

Los niveles de funcionamiento cognitivo

Comencemos con una síntesis de los estadios del desarrollo cognitivo tal como podrían derivarse del trabajo original de Piaget, en la medida en que afectan a este artículo, es decir, en los niveles correspondientes al niño en edad escolar, para ocuparnos después de algunos desarrollos más recientes, antes de volver a la relación específica a las variables en juego en el aprendizaje de las matemáticas. Los cinco estadios con los que tendremos que ver se han denominado:

(1) pre-operatorio (4-6 años)

(2) temprano de operaciones concretas (7-9 años)

(3) medio de operaciones concretas (10-12 años)

(4) último de operaciones concretas (formal temprano o de generalización concreta) (13-15 años)

(5) de operaciones formales (16 años en adelante).

Pero avisemos de entrada que las edades croniológicas correspondientes a los estadios varían mucho de una a otra cultura, de una a otra persona y, de hecho de una a otra tarea en la misma persona: es el orden de sucesión de los estadios lo que permanece invariante. Los niveles de edad empleados en este artículo se ads- 
criben a cada estadio sólo para orientar al lector respecto al nivel de curso aproximado de los niños en que el nivel en concreto de funcionamiento que se describe se encuentra con mayor frecuencia. Más aún, los estratos de edad no se refieren al nivel medio de funcionamiento cognitivo de los niños, sino más bien a la capacidad de pensamiento óptima en un período dado de su desarrollo. Es importante destacar que cualquier individuo puede operar en cualquiera de estos niveles según la situación. Los adolescentes pueden dar, y de hecho dan, respuestas que pueden clasificarse como preoperatorias. En la mayoría de los niveles de edad, desde el de primaria superior hasta el de secundaria superior, pueden encontrarse ejemplos de cada uno de los niveles operatorios. Naturalmente, las respuestas en niveles superiores se dan con mayor frecuencia en los últimos años de la escuela secundaria. También ayudan a determinar el nivel en que operará el estudiante la naturaleza de la tarea y la familiaridad del estudiante con la tarea.

\section{Estadio pre-operatorio}

El pensamiento en el estadio pre-operatorio es lógicamente limitado, puesto que las dimensiones del problema aún no están diferenciadas. La percepción que tiene el niño de la situación es una unidad global rígida. Su razonamiento está dominado por el contexto perceptivo y encuentra difícil apreciar las relaciones y establecer juicios consistentes. Así, su razonamiento no tiene por qué estar orientado a la realidad, sino que puede reflejar simplemente una asociación personal y peculiar de su propia experiencia. El pensamiento pre-operatorio refleja lo que Piaget ha denominado "procesos transductivos" en los que el niño establece una asociación desde lo particular hacia lo particular. Estas asociaciones transductivas son totalmente arbitrarias y conducen habitualmente a conclusiones erróneas. Por ejemplo el niño puede tomar un elemento destacado de un acontecimiento y pasar después irreversiblemente a concluir a partir de él algún otro acontecimiento perceptivamente dominante que el niño ha relacionado con aquél a partir de su experiencia.

En un experimento muy conocido que ejemplariza el razonamiento en el nivel pre-operatorio, se echa limonada en dos vasos exactamente idénticos hasta la misma altura. El niño asiente a que ambos son iguales. Se echa entonces la limonada de un vaso en otro vaso más alto y estrecho y alcanza, por supuesto, un nivel superior. El niño preoperatorio elige, casi invariablemente, el vaso alto, "porque tiene más». «¿Por qué?» «Porque es más alto». Cuando la limonada del vaso alto se echa en un vaso ancho y aplastado, el niño pasa a preferir el original, "porque ahora es más alto y tiene más». El niño pre-operatorio fundamenta sus juicios en las apariencias y en las impresiones totales. No puede ver los componentes de la situación y que, aunque pueda elevarse la altura del líqui- 
do, el área de corte del cilindro de líquido se ha reducido proporcionalmente. Su pensamiento en este experimento es típico del estadio, unidimensional. No deja de tener mucho interés el que esto baste con frecuencia para emitir un juicio correcto: después de todo, un tren más largo tiene también, la mayoría de las veces, más vagones. En el caso que hemos presentado, existe un número muy limitado de dimensiones obvias, y ocurre que la que han seleccionado es una de ellas. Si se complica un poco el problema, la respuesta se liga típicamente a una irrelevancia. En cualquier caso, aquí lo esencial es que las tareas de dos dimensiones, en las que es preciso relacionar una tarea con la otra, están más allá de la capacidad del niño pre-operatorio.

\section{Niveles operatorios concretos}

Los procesos de pensamiento en los niveles operatorios concretos, forman un sistema limitado pero integrado que está ligado a las experiencias empíricas del niño. El niño que opera en estos niveles es capaz de aislar y de pensar cierto número de dimensiones de la situación de un problema, pero todavía no es capaz de imaginar acontecimientos o posibles resultados que no guarden relación con sus experiencias. El término "concreto" se emplea aquí para referirse a la idea de que las estructuras del pensamiento del niño dependen de la anterior experiencia, actual o reciente $\mathrm{y}$, de este modo, el pensamiento va ligado a la realidad concreta del niño. Durante el período de las operaciones concretas, el niño desarrolla estructuras de pensamiento como parte de su desarrollo mental general, que le permiten clasificar materiales, desglosar grupos en subgrupos, colocar en orden una serie, emparejar elementos correspondientes y sustituir elementos equivalentes. Las capacidades para ejecutar estas operaciones completan un sistema lógico y capacitan al niño para formar conceptos que se relacionan directamente con su experiencia, pero no le capacitan para manipular relaciones entre abstracciones.

Varios investigadores han señalado un importante número de limitaciones del pensamiento circunscrito al nivel concreto. En primer lugar, los niños de este nivel no delimitan posibles contingencias de los resultados ni comprueben cuál de ellas se da de hecho en los datos presentes. En lugar de ello, estructuran y organizan la información presente en los datos sobre la base de lo que se da en el presente inmediato. En otras palabras, las estructuras mentales existentes están insuficientemente despegadas del objeto material a mano como para obtener una comprensión independiente del contęnido, de la estructura en cuestión. En segundo lugar, aunque los diversos sistemas de estructurar el pensamiento son adecuados para el área concreta particular desde la cual y para la cual fueron desarrollados, estos sistemas no son combinables en un todo unificado. $Y$ este último desarrollo es necesario para que el individuo sea capaz de manejar problemas complejos y multivariables, en el área. 
Debería quedar claro que los estadios de las operaciones concretas son preparatoriós. Los niños pueden manejar los problemas si se les brinda un apoyo concreto adecuado a su pensamiento. Pero si se les constriñe a depender de sus recursos simbólicos en lugar de apoyarse en la evidencia de sus propios ojos, oídos y manos, abandonarán su lógica y regresarán a los métodos preoperatorios de aproximación.

\section{Nivel operatorio formal}

El nivel operatorio formal es el estadio en que el pensamiento pasa a ser genuinamente abstracto. El pensamiento formal manifiesta la capacidad de establecer y comprobar hipótesis y de abstraer principios comunes a partir de datos o experiencias concretas. Los estudiantes que operan en el nivel formal pueden dirigir su atención a la forma de un argumento o situación e ignorar el contenido concreto. Mientras que el niño en el nivel de las operaciones concretas se remite fielmente a la realidad (el niño preoperatorio ni siquiera hace eso, sino que colorea la realidad con una percepción egocéntrica de la situación), el pensador formal es capaz de ir un paso más allá y de anticipar las posibles formas que pueda tomar la realidad.

Un ejemplo ampliamente utilizado que ilustra las cualidades del pensamiento formal en cuanto distinto del concreto, es el problema del descubrimiento de las relaciones entre los factores que influyen el vaivén o período de un péndulo. Se proporciona al niño un cronómetro, cuerda de distintas longitudes, una regla y un peso ligero y otro pesado. Los niños que operan en un nivel concreto son capaces de observar que el empujón inicial no afecta al período del balanceo. Examinan a continuación otros factores: la pesadez del peso y la longitud de la cuerda. Para probarlos pueden atar un peso ligero a una cuerda corta y cronometrar el vaivén, atar después un peso pesado a una cuerda larga y cronometrarlo. Puesto que el primero de ellos se moverá más deprisa, llegarán a la conclusión de que el período depende del peso y de la longitud de la cuerda. Son capaces de tomar nota de la causa y el efecto en ejemplos concretos, pero parecen incapaces de controlar y comprobar sistemáticamente todas las variables. Para poder establecer la relación correcta es necesario controlar el peso con independencia de la longitud y la longitud independientemente del peso. Es preciso que el niño vea que se dan cuatro posibilidades y no dos. En el nivel de las operaciones formales el niño es capaz de estructurar su experimento con un diseño similar al de la siguiente tabla, y extraer la conclusión correcta.

$\begin{array}{lll}\text { Peso } & \text { Longitud } & \text { Resultado } \\ \text { pesado } & \text { largo } & \text { lento } \\ \text { ligero } & \text { largo } & \text { lento } \\ \text { pesado } & \text { corto } & \text { rápido } \\ \text { ligero } & \text { corto } & \text { rápido }\end{array}$


El punto importante es que el planteamiento operacional formal concibe la realidad no sólo en términos de lo que es, sino también en términos de lo que podría ser. En el pensamiento formal, las dimensiones del problema se abstraen y combinan lógicamente, llegando así a su resultado que podría no haber sido de hecho observado o no ser relacionable con la experiencia pero que es lógicamente posible. De este modo, el pensamiento formal es más flexible, se encuentra libre de las restricciones de la realidad inmediata y puede, por tanto, oscilar hacia atrás y hacia adelante según consideramos puramente lógicas.

\section{Estructura de las respuestas del niño}

La última parte del artículo incluye un breve resumen de las características del funcionamiento cognitivo del niño en distintos estadios de desarrollo. Originalmente se derivaron de la observación cuidadosa de las respuestas infantiles a tareas que implicaban conceptos lógicos. El modo en que los sujetos estructuraban y reestructuraban (cuando era conveniente), sus respuestas, fueron la clave para definir los estadios. Se tuvo cuidado en observar el proceso en marcha, mientras que el acierto o incorrección de la solución final era en gran parte irrelevante. Esta investigación llevó a una definición de lós estadios de desarrollo que supone unas características relativamente estables para un individuo en un determinado período. Pero esto en sí no es de gran ayuda para los educadores a los que interesa el desarrollo de la capacidad del niño para manejar conceptos en áreas determinadas del curriculum. Parecería que simplemente proporciona etiquetas globales del tipo de «razonador formal", «razonador concreto", etc., que pueden ser gravemente engañosas. Los maestros saben por ejemplo que un estudiante puede dar respuestas característicamente formales en matemáticas y al tiempo dar respuestas concretas de un bajo nivel relativo en literatura inglesa. Lo que parece precisarse es un método general para analizar las respuestas tal como se dan en distintas áreas de contenido. Al llegar aquí hay que subrayar que no es la corrección de la respuesta final lo que cuenta, sino la estructura de razonamiento que la generó.

En un trabajo reciente (Collis y Biggs, 1977) sobre este problema, se ha encontrado útil interponer terminología relativa a la descripción de la respuesta entre el estadio base de desarrollo cognitivo y las características de la respuesta, e introducir el concepto de espacio de la memoria actuante. La tabla.1 resume los aspectos clave de aquellos conceptos que son destacadamente útiles para el análisis de muchas tareas escolares, incluidas aquellas que afectan a las matemáticas.

Examinemos en primer lugar la idea de capacidad de la memoria actuante. En el presente contexto se refiere a la cantidad de espacio disponible para mantener los elementos y operaciones relevantes en el primer plano del «ojo de la mente» mientras lleva 
a cabo el trabajo necesario para dar una respuesta. Por supuesto, los elementos y operaciones pueden provenir de inputs sensoriales directos, del almacén de la memoria a largo plazo o en parte de cada uno de ellos. Se ha propuesto que el grado de capacidad disponible varía a diferentes niveles de funcionamiento y bajo diferentes condiciones. Case (1977) sostiene este punto de vista y, en un interesante artículo teórico apoyado por evidencias de investigación, desarrolla mucho más esta idea. Sugiere que la progresión dentro de cada estadio depende del incremento en la capacidad de la memoria actuante, que a su vez resulta del incremento en la automaticidad de las operaciones básicas de cada estadio. Esto se ajusta a mi propia experiencia (1975a, b) que muestra que en todo su transcurrir, desde las operaciones concretas más tempranas hasta la generalización concreta, el desarrollo se caracteriza por una progresión de estrategias ejecutivas cada vez más complejas y potentes que permiten no sólo que puedan acomodarse cada vez más y más datos, sino también ir manejando más y más operaciones concretas.

El punto de vista de Case de que se va desarrollando y conformando dentro de cada estadio un determinado conjunto básico de operaciones, es interesante y está también apoyado por datos procedentes de los items de tipo matemático. Por ejemplo, a lo largo de los subestadios que constituyen el período de las operaciones concretas, aparece un desarrollo gradual de los elementos básicos de la lógica elemental, a lo largo y desde lo que podría denominarse lógica clasificatoria, hasta la generalización concreta. Finalmente Case señala que un determinado grado de automatización en las operaciones básicas del estadio previo es un prerrequisito para el desarrollo en el estadio siguiente. En otras palabras, el avance hacia el razonamiento de las operaciones formales, con sus requisitos de pensamiento con abstracciones y comprobación de hipótesis, dependería de que los indivíduos hubieran automatizado las operaciones básicas del último estadio concreto: la capacidad para manejar variables, por ejemplo, iría precedida de una competencia para trabajar con números generalizados (Collis 1975b). Por ejemplo, en el nivel de generalización concreta los adolescentes encuentran natural la idea de que la fórmula $\mathrm{V}=\mathrm{L} \times \mathrm{B} \times \mathrm{H}$, donde $\mathrm{V}$ representa el volumen de un prisma recto y $\mathrm{L}, \mathrm{B}, \mathrm{H}$ tienen las connotaciones habituales en este contexto, daría una medida correcta del volumen para una multitud de prismas rectos, en.la medida en que sustituyéramos los números que aparecieran en cada fórmula. Pero hasta el estadio de las operaciones formales no son capaces de abordar directamente cuestiones relativas a las relaciones existentes una afirmación tal como: si «B» se duplica y «L" permanece igual, ¿qué debe hacerse $\mathrm{a}$ « $\mathrm{H}$ " para mantener constante $a$ « $V$ »?

Al llegar a este punto de la discusión parecería útil ilustrar los tipos de respuestas conductuales con que nos encontramos cuando los niños exceden su capacidad para manejar el material. En un 
estudio (Collis, 1975a) se halló que los niños en el nivel más temprano de las operaciones concretas eran claramente capaces de trabajar significativamente con items matemáticos elementales que implicaban dos elementos y una operación (por ejemplo, $2+3$ ) pero eran incapaces de trabajar con éxito si se introducian un elemento y una operación adicional (por ej. $2+3+4$ ). En el siguiente nivel de las operaciones concretas (medio superior), los niños tenían ya éxito en este último item. Quizá, desde la peršpectiva de la teoría de Case, esto ocurría a causa de que una experiencia más extensiva y prolongada con items del primer tipo conducía a la suficiente automaticidad de la operación requerida como para liberar más espacio de trabajo en la memoria para poder hacer frente a las exigencias extra de los items del segundo tipo. Bien sea ésta o no la explicación correcta; el protocolo típico del niño en el nivel más temprano de las operaciones concretas tenía esta línea:

$E$ : ¿A qué número es igual $2+3+4$ ?

$S: 2+3=5$ y (pausa)... ¿cuál era el otro número?

$E$ : He dicho ċa qué número es igual $2+3+4$ ?

S: iAh sí! Entonces, 2 más (pausa)... cंcómo era la suma?

En el siguiente nivel de las operaciones concretas se demuestra un fenómeno similar si aumentamos el nivel de abstracción de los elementos implicados: grandes cifras, por ejemplo y el número de las operaciones a controlar. A continuación tenemos un protocolo de uná niña de 11 años recogido en video-tape por el autor:

$E$ : Si te dicen que

$$
\left.\begin{array}{l}
y=365 \\
y+489=489+.365
\end{array}\right\} \text { escrito en la pizarra }
$$

¿Puedes decirme el valor de (y))?

$S:$ Creo que sí cipuedo hacerlo en la pizarra?

$E$ : Por supuesto (le ofrece tiza)

(Se animaba a $S$ a hablar en voz alta a medida que pensaba y trabajaba). $S$ se movía hacia el encerado e indicaba que iba a "sumar 489 y 365)). Y lo hizo, así,

$$
\begin{array}{r}
489 \\
365 \\
\hline 854
\end{array}
$$

Volvió a continuación a la proposición y $+489=489+365$ y, frun ciendo el ceño leyó en voz alta la proposición completa como inten. tando grabarla en su memoria. Echaba al mismo tiempo miradas ocasionales al trabajo que había hecho. De repente exclamó (ah)" y fue rápidamente hacia donde había estado trabajando al principio, con la 
TABLA $\cdot 1$

Estadio base de desarrollo cognitivo y descripción de respuestas

Estadio base

de desarrollo cognitivo

Edad de respuesta

Operaciones formales

16

Abstracta extensa

Máxima

Capacidad de

la memoria

de trabajo

Clave
Descripción diagramática de la respuesta

Selección de posible Respuesta respuesta

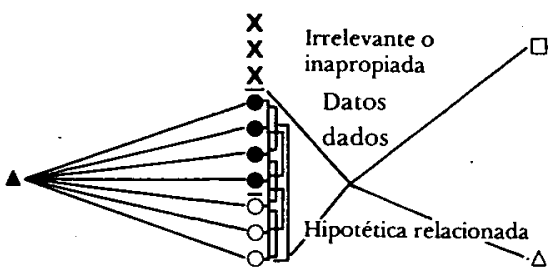

Generalización concreta $13 \cdot 15$ Relacional

Alta

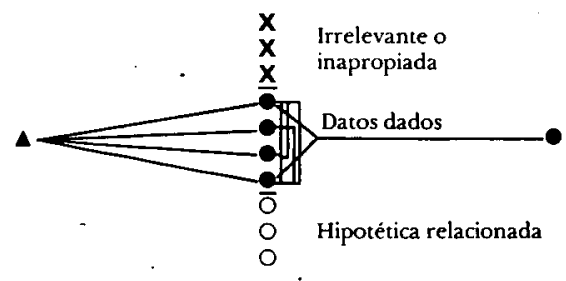

Concreto medio

$10-12$ Multi-estructural

Media

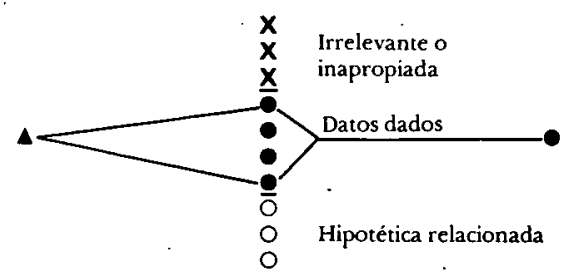

Baja

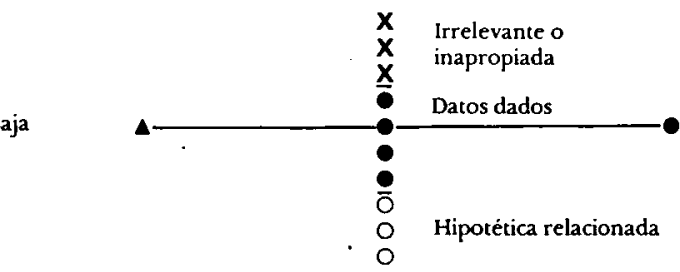

\section{Concreto temprano}

Uni-estructural

Preoperatorio

4. 6 Pre-estructural

Mínima

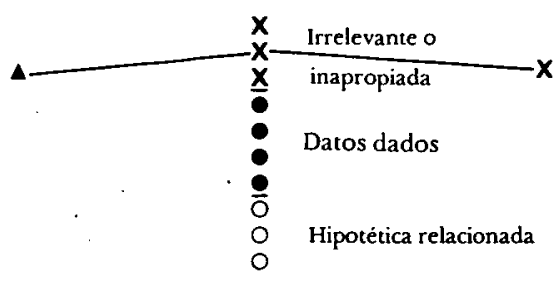


tiza preparada, para empezar otra vez a trabajar. Para cuando llegó - alli, (una fracción de segundo después) había olvidado aparentemente lo que iba a hacer y volvió a leer otra vez la proposición. Después de algunos movimientos más de un lado para otro se volvió a $E$ y dijo con voz resignada «No puedo hacerlo). Durante todo este tiempo $S$ mostraba signos externos de gran esfuerzo mental y, antes de abandonar, de un auténtico estrés.

En ambos casos, el problema que tenían los niños parecía estar estrechamente relacionado con una incapacidad para retener durante el tiempo suficiente para procesarla toda la información necesaria. En el segundo caso la niña parecía estar sobrecargada desde el principio por la cantidad de datos proporcionados: leyó el problema completo, pero ignoró completamente la importancia de la primera afirmación: $y=365$.

Los niños entrevistados situados en el siguiente nivel de las operaciones concretas (generalización concreta) no tuvieron problema con estos ejercicios, aunque mostraron el mismo tipo de conducta cuando se elevó a variables el nivel de abstracción de los elémentos y cuando se hicieron más complejas las operaciones.

Resumiendo, consideremos el diagrama de la Tabla 1 a la luz de lo que hemos dicho hasta aquí. Aunque estas representaciones diagramáticas de lo que ocurre entre el momento en que se da al niño la clave y su respuesta de hecho, se deducen en gran parte de la investigación y de la observación en otras áreas de contenido distintas de las matemáticas, (ver por ej. Hallam, 1967, 1969; Jurd, 1970; Mason, 1974; Peel, 1960, 1975; Rhys, 1966; Sutton, 1962, 1964). No es difícil ver su relevancia para el aprendizaje matemático.

La clave representa una cuestión o afirmación a la que se espera responda el sujeto y se extrae a partir de los datos facilitados. Los datos pueden ser una serie de proposiciones que quizá forman una historia o plantean un problema matemático. En este caso, se exponen ante el niño los datos reales con los que ha de trabajar. Otras veces, los datos pueden tomarse del almacén de memoria a largo plazo del sujeto. En este último caso la clave debe traer a la memoria los datos relevantes almacenados previamente por el sujeto.

Los símbolos en la columna de selección de respuesta representan proposiciones que pueden ir desde frases normales (Guillermo fue Rey) hasta proposiciones matemáticas $(2+3+4)$. Las líneas representan las conexiones implicadas en cada nivel de respuesta concreto. La columna final muestra los tipos de respuesta real típicos de cada nivel. Podemos ver que en todos los niveles, excepto en Abstracta Extensa, la respuesta real es precisa y definida: representa una decisión o cierre firme.

En el nivel pre-operatorio, en que el nivel de respuesta es típicamente pre-estructural, y donde la clave y la respuesta se confun- 
den con frecuencia, parece que el niño utiliza dos estrategias básicas como mínimo. La primera es el rechazo del problema, negándose simplemente a implicarse o mediante una tautología que reexpone el problema. La segunda consiste en el razonamiento transductivo. Es esta última estrategia la que se ilustra en el diagrama. El alumno intenta diferenciar una respuesta relevante, pero no lo consigue porque no tiene suficiente capacidad (y quizá experiencia) para formular una base lógica suficientemente comprensiva para seleccionar una respuesta relevante. En el razonamiento matemático, las respuestas de no conservación en problemas de número, longitud y cantidad, pueden considerarse ejemplos de este tipo de razonamiento. Por ejemplo, el niño que asiente a que hay el mismo número de huevos que de hueveras, cuando ambos se sitúan en filas en correspondencia uno-a-uno, pero que decide que hay más de uno que de otro componente cuando en uno de los conjuntos se modifica únicamente la longitud de la fila. Como explicamos anteriormente, en eso consiste el razonamiento transductivo cuyo fallo, en tales ocasiones, se debe a que el niño ha atendido sólo a un componente lógico de la tarea, cuando para la respuesta correcta se requiere tener en cuenta dos componentes al menos. En otras palabras, el niño no puede manejar la estructura matemática más elemental en que es preciso relacionar dos componentes totalmente visibles.

Puede verse que para los niños en el estadio de desarrollo preoperatorio, las matemáticas, tal como las definimos en la primera parte de este artículo, no constituyen un estudio que pueden abordar con posibilidades reales de éxito. Igualmente claro es que, si la experiencia es un elemento esencial para el desarrollo de superiores niveles de funcionamiento, el niño situado en este estadio necesitará tener numerosas experiencias prematemáticas y prácticas, con el fin tanto de adquirir experiencia en el campo en cuanto tal, como de desvelar las inconsistencias que surgen en el razonamiento transductivo.

La respuesta uniestructural, típica del estadio de desarrollo temprano de las operaciones concretas, representa la capacidad para concentrarse en determinados aspectos de los datos reales, seleccionar un aspecto relevante y responder inmediatamente sobre esta base. En áreas de contenido distintas de las matemáticas, el caso representativo habitual es que el niño da una respuesta precisa y relevante que se apoya en una pieza de evidencia tomada de los datos facilitados, siendo la pieza de evidencia seleccionada algún aspecto especialmente llamativo para el sujeto en ese momento.

En matemáticas este fenómeno se manifiesta en la capacidad para trabajar significativamente con operaciones simples sobre elementos concretos. Por ejemplo, y en lo que se refiere al sistema numérico, esto signífica la utilización de una de las cuatro opera- 
ciones de la aritmética elemental con números pequeños. La concreción de las operaciones la garantiza alguna analogía física (la adición relacionada con la unión de conjuntos disjuntos, por ejemplo), mientras que la concreción de los números la garantiza la disponibilidad del material físico. Aún con estas restricciones el niño ve la necesidad de "cerrar» la operación. Los niños de este nivel no tienen así ninguna base sobre la que decidir sobre la equivalencia de proposiciones tales como $3+2$ y $4+1$, excepto cerrando cada una de ellas por separado. Incluso el hecho de que ambos resultados dan "5" no capacita al niño para sacar la conclusión de que $3+2=4+1$. Cada uno de ellos es un juicio por separado que da la casualidad de dar $« 5 »$.

A este nivel del desarrollo podemos ver que el niño, una vez que ha alcanzado una serie de conservaciones básicas, necesita tener para trabajar significativamente con números, una amplitud de experiencias apropiada a este estadio del pensamiento. En este estadio los niños no tienen aún la capacidad para empezar a construir un sistema matemático en cuanto tal, pero observamos que ya empiezan a estar disponibles, en forma de estructuras concretas elementales, los ladrillos para sus cimientos. La experiencia con estas estructuras aumentará su automaticidad, por emplear la expresión de Case, lo que liberará espacio de trabajo en la memoria, de tal modo que pueda manejarse más de una operación a la vez.

Si pasamos a la respuesta multiestructural, típica del nivel medio de las operaciones concretas, vemos que el niño necesita todavía llegar a una decisión definida (clausura), pero que utilizará como base dos o más piezas de información relevante no necesariamente conexa. Esto se representa en el razonamiento matemático por la capacidad para enfrentarse a proposiciones equivalentes con números pequeños, mediante recombinaciones de los números cardinales de los conjuntos representados en la proposición. Por ejemplo, estos niños pueden decidir en esta etapa que $3+2=4+1$ sin recurrir a la clausura. Por su parte, si se hacen tan grandes los números que escapen a visualización física, por ejemplo, $789+326=790+325$, el niño se repliega a la clausura, es decir, cierra cada parte por separado y compara.

Por añadidura, la capacidad demostrada por la respuesta multiestructural posibilita que se abra camino el sentimiento de que es necesaria una consistencia. Esta sensibilidad hacia la consistencia (Collis, 1975a) sin haber sido capaz de justificarla lógicamente, capacita en este nivel al niño para comenzar a desarrollar sistemas matemáticos simples, (un sistema de co-variaciones con fundamento concreto) y representa de este modo un nivel de desarrollo en el que ya pueden comenzar a manejarse las matemáticas en cuanto tales (tal como las definíamos al principio). Es probablemente en este estadio del desarrollo cuando empieza a tener utilidad para el programa escolar de matemáticas el comenzar a desa- 
rrollar una estrùctura concreta de experiencias que pueda ir construyéndose año tras año para formar un sistema lógico concreto isomórfico con las matemáticas abstractas formales.

El desarrollo que se evidencia a continuación en forma de respuesta, afecta al nivel relacional. Las respuestas que se dan en este nivel, típico del niño con capacidad de generalización concreta, demuestran que el sujeto ha tenido en cuenta todos los aspectos de los datos y las interrelaciones implicadas entre ellos. Este nivel proporciona un concepto o principio general, aunque se mantiene firmemente dentro del marco, concreto, exigiendo. que se llegue a una clausura. La capacidad que hemos mencionado en último lugar resalta una de las'debilidades básicas de este nivel de funcionamiento, la necesidad de clausurar, o de llegar a una decisión, que genera una tendencia a generalizar sobre los escasos ejemplos específicos proporcionados en los datos.

Los niños cuyas respuestas en matemáticas se sitúan en este nivel, pueden manejar lo que pueden presentarse como conceptos abstractos, esto es, elementos como variables que no tienen contacto directo con su realidad física, pero que, una vez investigados resultan ser números generalizados. El niño puede manejar cierto número de operaciones, incluso masivamente, no asequibles físicamente, números, en la medida en que tiene una garantía de que los elementos y sus combinaciones pueden clausurarse en cualquier momento y proporcionar un resultado único que puede ser aplicado a la realidad física.

La capacidad para concretarse en las interrelaciones entre las proposiciones insertas en los datos permite al sujeto la búsqueda de una consistencia dentro del sistema concreto implicado. Típicamente, el sujeto busca una consistencia dentro de sistemas simples con una base concreta. Puede verse que incluso dentro de las constricciones implícitas en la anterior descripción, el sujeto es capaz en este nivel de desarrollar toda una estructura matemática compleja en la medida en que tenga un fundamento concreto. Pueden utilizarse operaciones matemáticas múltiples en diversas combinaciones con la limitación de que deben ser susceptibles de clausura en algún momento. Los elementos, así como sus símbolos, presentan escasa dificultad, a menos que se internen en el dominio de lo abstracto y carezcan de una contrapartida físicamente observable. Esto significa que se les escapan los conceptos que implican nociones abstractas de razón y proporción, pero que, si se les proporcionan fórmulas en que estén incluidas estas nociones, son capaces de emplearlas para llegar a resultados concretos.

El nivel de respuesta abstracto ampliado representa un avance importante que se produce una vez desarrollada la capacidad lógica, en el estadio de las operaciones formales. Típicamente este nivel de respuesta tiene en cuenta, no sólo todas las interconexiones en los propios datos facilitados, sino que busca relaciones en- 
tre estos y proposiciones y estrategias hipotéticas que, aunque no se hayan facilitado, se sugieren por los datos como lógicamente apropiadas. Es característico de este estadio el que los alumnos se complacen en la comprobación de hipótesis y poseen la capacidad de mantener abiertas un gran número de posibilidades mientras examinan sus consecuencias. Evitan una decisión o clausura inmediata mientras examinan las posibilidades, no necesariamente contrastándolas con la realidad, sino en términos de la estructura lógica de las propias proposiciones. A juzgar por los materiales recogidos, el resultado de este procesamiento es con más frecuencia una solución(es) cualificada(s) que una decisión bien definida.

Al trabajar con material matemático, el alumno queda liberado de la necesidad de relacionar elementos, operaciones, o la combinación de ellos, con análogos físicos, y puede tomar como realidad un sistema abstracto bien definido con sus definiciones, relaciones y reglas: no se aborda la clausura hasta que no se han probado todas las posibilidades. En este nivel los alumnos, debido a su capacidad para tener en cuenta constructos hipotéticos relacionados, trabajan casi automáticamente con las situaciones o condiciones limitantes y pueden así operar con problemas que implican cálculo elemental o cálculo de lugar geométrico. Tienen una exigencia por la consistencia en cualquier sistema establecido, ya sea simple o complejo, concreto o abstracto y pueden reconocer (y evitar) cualquier inconsistencia.

Estos alumnos tratan las proposiciones matemáticas como relaciones interdependientes entre variables que pueden ser manipuladas operando dentro de las reglas inherentes al sistema, tal como haya sido éste definido. Manejan muy bien los elementos. abstractos, variables y operaciones. Como parte de su capacidad para manejar las operaciones, tenemos un ejemplo válido en el uso que hacen de la inversión. Ven esta operación como si trabajasen directamente sobre la operación afectada, de tal modo que se equilibre o compense sin afectar necesariamente a la existencia de la operación anterior o se corra peligro de trastocar las relaciones originales existentes en la proposición. En suma, es razonable sugerir que los alumnos que se encuentran en este estadio de desarrollo están listos para trabajar con el sistema formal abstracto de estructura que, para el matemático, constituye la esencia de las matemáticas.

Nos hemos ocupado hasta aquí de los sujetos cuyo nivel de respuesta está limitado por su capacidad cognitiva tal como se representa en un estadio particular de desarrollo. Aunque sea brevemente, debemos considerar ahora a las personas que demuestran tener la capacidad cognitiva de responder a un nivel más alto pero que, en un área específica o en una situación concreta, responden a un nivel mucho más bajo que el que su capacidad aparente parecería indicar. Wason (1974) describe este fenómeno desde el punto de vista del investigador cuando señala que muchos de los erro- 
res cometidos por académicos muy inteligentes cuando intentan resolver su "problema de los cuatro naipes", se equiparan a las respuestas que cabría esperar de personas que operan en el nivel de las operaciones concretas. Podemos observar este fenómeno en actividades cotidianas cuando la persona en cuestión intenta trabajar en un área con la que tiene poca familiaridad. El profesional altamente cualificado, como el abogado o el médico que intenta resolver algún problema mecánico en su coche, puede regresar a respuestas típicas de los estadios de funcionamiento más bajos. Se descubre con mucha frecuencia en estos casos que la persona en cuestión no ha tenido sufiente experiencia en el área como para ser capaz de seleccionar las variables relevantes que es preciso procesar. Dispone de la capacidad operatoria, pero no tiene los datos con los que operar.

Los comediantes suelen utilizar deliberadamente este fenómeno. Muchas historias de humor parecen depender de contemplar a alguien cuya aparente capacidad de funcionamiento cognitivo es alta, colocado en una situación extraña en que su conducta indica un nivel mucho más bajo de respuesta del que cabría esperar. Los chistes étnicos se basan con frecuencia en señalar que el grupo minoritario responde a un nivel pre-estructural.

Estas observaciones tienden a apoyar la tesis de Piaget de que la experiencia en un universo concreto del discurso, es un prerrequisito esencial para responder a un nivel alto en tal área. Tiene también importancia el punto de vista de Case de que es necesaria una cierta automaticidad de las operaciones básicas típicas de un nivel de desarrollo cognitivo para liberar espacio de trabajo en la memoria, y posibilitar un avance hasta el próximo nivel. Son claras las implicaciones de estos aspectos para la enseñanza de las matemáticas a cualquier nivel. Antes de que podamos esperar que los alumnos respondan a un nivel superior en un área concreta, hay que proporcionarles suficientes experiencias en las que sea adecuado un nivel de funcionamiento inferior, para que puedan familiarizarse con la idiosincrasia del área y automatizar alguno de los constructos y/o operaciones básicos. Este principio de enseñanza no es nuevo y ha sido utilizado intuitivamente por generaciones de buenos maestros. Pero hoy, sin embargo, tenemos algún criterio de razón para elegir las estrategias, así como alguna base para juzgar los tipos de experiencia apropiados y el grado de facilidad requerido antes de que podamos esperar que el alumno sea capaz de responder al nivel superior.

Podemos tomar como ejemplo la enseñanza de la teoría de Conjuntos a.los alumnos cuyo estadio de desarrollo cognitivo podemos asumir que está al nivel de las operaciones formales. Si el tema es nuevo para los alumnos, los intentos de arrancar inmediatamente con teoremas y definiciones formales abstractas dejarán a la mayoría enredados y haciendo analogías inadecuadas y ad 
hoc con otros temas que hayan seguido en su trabajo anterior. Sus niveles de respuesta serán típicamente multiestructurales o relacionales. Pero si el maestro dedica suficiente tiempo a introducir el tema a través de ejemplos concretos como las rotaciones de diversas formas geométricas, es muy probable que consiga que la mayoría de los alumnos respondan al nivel abstracto extenso. Esta introducción al tema permite que el alumno lo sitúe en un contexto, ayudándole a conceptualizar las nuevas operaciones, símbolos y estructuras y a ver donde se ajustan respecto a su estructura matemática ya existente. Una vez logrado ésto, el alumno, que tiene ya la capacida para el razonamiento operatorio formal, debería ser capaz de pasar rápidamente al sistema abstracto que implica el uso de definiciones y teoremas.

El desarrollo cognitivo en relación con algunos conceptos matemáticos

Para concluir este examen de la relación de la teoría de Piaget del desarrollo cognitivo con el aprendizaje de las matemáticas, parecería pertinente tomar algunos conceptos matemáticos específicos y examinarlos respecto al tipo de items que pueden manejar los sujetos en los distintos estadios de desarrollo. Los datos experimentales sobre los que fundamentamos este apartado pueden encontrarse en dos de las anteriores publicaciones del autor, Collis (1975a y 1975b). Los conceptos seleccionados están relacionados directamente con aquellos establecidos en el apartado anterior de este artículo y que describían la naturaleza de las matemáticas elementales. La capacidad observada en el niño para manejar estos conceptos, permite hacer deducciones sobre los estadios en el desarrollo cognitivo y tiene también implicaciones directas para los curricula escolares de matemáticas y para los métodos de enseñanza de la materia. Hemos seleccionado para la discusión . los siguientes conceptos:

(1) Números y operaciones.

(2) Combinación y operaciones.

(3) Sustitución pronumeral.

(4) Operación inversa.

(5) Conclusión.

(6) Sistemas matemáticos.

\section{NUMEROS Y OPERACIONES (Collis, 1975a, b)}

\section{Estadio de las primeras operaciones concretas}

Ya indicábamos en un epígrafe anterior que el niño, en el estadio temprano de las operaciones concretas parece necesitar te- 
ner la operación clausurada de hecho y con un resultado único, para que tal operación tenga sentido para él. Incluso se siente más a gusto si se halla implicada una sola operación y ésta puede relacionarse estrechamente con operaciones físicas que intervienen en el proceso de un determinado cambio físico. En este estadio, el niño puede manejar con sentido items aritméticos que suponen la realización de una clausura, aun cuando esto exija llevar a cabo una comparación con un resultado dado puede por ejemplo resolver problemas de este tipo:
(a) Si $q=8+4$ entonces $q=$ ?
(b) $4+3=$ ?
(c) Si $7 * 4=3$ entonces $*=$ ?

Con números pequeños, que parecen usar cómodamente los niños de este estadio, los items como (c) no parecen presentar más dificultad que los items como (a) y (b).

\section{Estadio medio de las operaciones concretas}

En este estadio el niño es capaz de trabajar con números grandes formando parte de operaciones sencillas y con cierto número de operaciones en secuencia si los números se mantienen pequeños. Parece necesario, sin embargo, que sea capaz de clausurar cáda operación en secuencia para poder tener el conocimiento seguro de que el resultado de la secuencia de clausuras proporciona un resultado único. Algunos ejemplos de los tipos de item que puede manejar un alumno que opere a este nivel son:
(a) Si $x=475-234$
entonces $\mathrm{x}=$ ?
(b) Si $378 * 231=147$
entonces * $=$ ?
(c) $\mathrm{n}=(6 \times 8) \div 4$
$\mathrm{n}=$ ?
(d) $(3 * 6) \div 3=6$
$\pi=$ ?
(e) $(2 * 3) * 4=9$
$*=$ ?
(f) $5 * 3=4$ ó 2
$*=$ ?

Puede verse que el alumno que opera en el nivel medio de las operaciones concretas puede manejar dos clausuras en secuencia y que esto no le apura cuando ha de hacer tanteos, como en los ejemplos (d), (e) y (f). Puede manejar en cambio números grandes si se trata de una operación única.

\section{Estadio de la generalización concreta}

En este nivel el alumno puede utilizar ya elementos generalizados (grandes cifras y pro-numerales) y hacerlo con sentido, además de haber generalizado lo bastante la idea de operación, hasta 
el punto de que ya no necesita cerrar cada operación mientras trabaja con ella. Aún necesita la garantía de la clausura, pero esto no significa que necesite cerrar secuencialmente operación por operación. En este estadio, la experiencia del alumno con los números y la fiabilidad de las cuatro operaciones aritméticas utilizadas en conjunción con ellos, parecen ser suficientes. Tiene capacidad para abordar items como los siguientes:
(a) $(4 \circ 3)$ o $1=5$ o $(1 \circ$ o 2$)$
$0=$ ?
(b) $(3$ o 4$)$ o $1=12 *(6 * 2)$
$0=$ ? $y^{*}=$ ?
(c) $(96 \times 42) \div 100=(96 \times 21) \div 50$ ¿Es esta proposición verdadera o
falsa?

\section{Estadio de las operaciones formales}

El alumno que funciona en el nivel formal demuestra un nivel de funcionamiento completamente nuevo. En este nivel la clausura es considerada, en el sentido matemático, como un fenómeno que hace posibles determinadas cosas: ya no se necesita la tranquilidad que proporcionan los números y las operaciones familiares. Pueden resolverse los problemas en que las letras representan números, o variables que empleen una operación bien definida. El alumno en el nivel de las operaciones formales, teniendo ya la capacidad para examinar la estructura de los items que le viene de no precisar obtener inmediatamente un resultado único, puede ahora considerar la posibilidad de más de una respuesta a un item. Los problemas como el que viene a continuación presentan poca dificultad en este nivel.
(a) $(\mathrm{a} \circ 3) \circ 4=8$
$0=$ ? y $\mathrm{a}=$ ?
(b) $7 * 6=5 * 4$
¿Es esta proposición verdadera o falsa?
(Dar una definición de la operación *.)

\section{COMBINACIONES DE OPERACIONES (Collis, 1975b)}

En un estudio diseñado para examinar con más detalle el desarrollo de la capacidad del niño para manejar operaciones aritméticas, se plantearon a alumnos (de 10 a 15 años), cuestiones que suponían hallar una operación (por ej. [6 $\left.{ }^{*} 2\right]^{*} 2=2,{ }^{*}=$ ?): Se pensaba que había menor probabilidad de que les hubieran enseñado cuestiones de este tipo, además de que las respuestas de los alumnos deberían revelar cómo veían ellos las operaciones aritméticas. Se aplicaron los criterios siguientes para diseñar las cuestiones:

(i) se utilizaron números pequeños (por ej., cifras menores de 20) para evitar la interacción que pudieran tener los números mayores con las operaciones. 
(ii) no estaban implicadas más de dos operaciones en ningu-

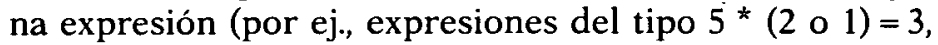
${ }^{*}=$ ?, $\mathrm{O}=$ ?).

Se examinó a los alumnos tanto individualmente como en grupo, de modo que pudiera registrarse cómo pensaban.

\section{Estadio medio de las operaciones concretas}

Los alumnos (aproximadamente de 10-11 años) que operan en este nivel, podrían resolver fácilmente problemas de "dos clausuras de una operación en secuencia», por ejemplo $(6 * 2) * 2=2,=$ ? y podrían determinar correctamente la operación en que fuera necesario hacer dos clausuras sencillas e independientes, por ejemplo, $8 * 2=11 * 5$, = Aún más, al hacerles, intentar operaciones como las que vienen en el ejemplo a continuación, estos alumnos podían deducir el valor de un número desconocido.

Fijate en esta proposición

$3 * 4=6 * a$

Si esta proposición es verdadera, entonces (puede haber más de una respuesta correcta).

* podría ser $\times$ sí no no sé

" podría ser $\div$ sí no no sé

" podría ser + sí no 'no sé

" podría ser - sí no no sé
En caso de (sii), entonces a debe ser

En caso de (sii), entonces a debe ser

En caso de (sí), entonces a debe ser

En caso de (sii), entonces a debe ser

En items en que se hallaban involucradas dos operaciones distintas, la estrategia que parecían utilizar los alumnos que operaban al nivel medio de las operaciones concretas era la de clausurar una operación y luego, o bien reconocer un modelo numérico que podría resolver el problema, o bien acometer una serie de ensayos para la segunda operación hasta lograr obtener un modelo. Por ejemplo, para responder a la cuestión $10^{*} 4=2$ o $3,{ }^{*}=$ ?, $0=$ ?, los alumnos tendían a trabajar de la siguiente manera:

Si el alumno supone ( $(+)$ para ( $(*)$ obtiene $14=2$ o 3 , lo que no le proporciona ningún modelo numérico, de modo que desencadenará en 
este término 2 o 3 , un par de ensayos, por ejemplo $2+3,2 \times 3$. Sabemos, sin embargo, que $2 \times 3$ con $10 * 4$ nos sugiere ( 6 ) etc.

Este método nó es sistemático ni muy fiable, puesto que depende demasiado y con demasiada rapidez del primer modelo que aparezca en los datos. No discernimos ningún intento de registrar. los ensayos ni de eliminar las posibilidades, y el hallazgo de la solución parecía ser en muchas ocasiones totalmente fortuito.

Uno de los resultados más interesantes de este estudio, fue el de ver la general incapacidad de los.alumnos que operaban en el nivel medio de las operaciones concretas para resolver items como:

$$
\begin{aligned}
& \text { (i) } \mathrm{Si}(4 \circ 2) \circ 3=6 \circ 4 \\
& \text { y a o }(3 \circ 6)=4 \circ 9 \\
& \text { hallar } \mathrm{a},
\end{aligned}
$$

y eso, a pesar de que podian en muchos casos resolver con éxito items del tipo:

$$
\text { (ii) }(12 \circ 3) \circ 2=8 \circ 4,0=
$$

y

(iii) Fíjate en esta proposición

$$
\text { (140.1) } \circ 1=10 \circ 5
$$

Si esta afirmación es cierta, entonces (puede haber más de una respues. ta corrécta)

o podríaser + si no no sé

En caso de ('sí) entonces a debe ser

o podríaser - si no no sé

En caso de (sí) entonces

a debe ser

- podríaser $\times$ sí no no sé

En caso de (si)) entonces

a debe ser

o podría ser $\div$ sí no no sé

En caso de ('sí) entonces a debe ser

La explicación que mejor ajusta parece ser la de que el problema reside en la diferencia entre proporcionar la $(s)$ operación (es) al niño para realizar las clausuras y hallar así «a» (como en el problema iii) y forzarle a deducir la(s) operacion(es) (como en el i) que han de utilizarse. Unicamente al llegar al estadio de la generalización concreta mostraron los alumnos capacidad para manejar items de este tipo (i). Los items en los que tuvieron éxito los alumnos en el nivel medio de las operaciones concretas fueron aquellos en que puede considerarse a la última parte del proceso como independiente de cualquier proceso inicial. Los alumnos fueron capaces de realizar un par de clausuras discretas y de comparar los resultados sin que una clausura estorbara a la otra. 
En contraste con los alumnos del nivel medio de operaciones concretas, los alumnos que operaban en el nivel de la generalización concreta (13-14 años aproximadamente) podían manejar con éxito la mayoría de los items del tipo:

$$
\begin{aligned}
& \text { Si }(4 \circ 2) \circ 3=6 \circ 4 \\
& \text { y a } \circ(3 \circ 6)=4 \circ 9 \\
& \text { hallar } a
\end{aligned}
$$

También era en este nivel donde los alumnos comenzaban a lograr respuestas correctas en items que implicaban dos operaciones distintas encerradas en la secuencia única (por ejemplo [ 4 * 3 ] o $2=2,{ }^{*}$ ?, $0=$ ?) o secuencias de clausuras requeridas en ambas partes de la ecuación (por ejemplo [12 o 3] o $2=8$ o 4). Los elementos que tienen én común para el éxito parecen ser, la capacidad para evitar que un resultado alcanzado en la primera parte de la secuencia de operaciones interfiera con la parte siguiente que se ha de procesar. Para comparar por ejemplo las diferencias de pensamiento entre los álumnos que operan en el nivel concreto medio y alumnos que operan en el nivel de la generalización concreta, observemos cómo tendían a trabajar los alumnos en el nivel de la generalización concreta.

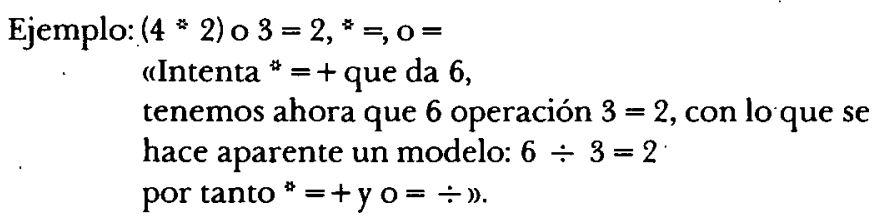

Las diferencias en la estrategia seguida en este estúdio por los dos grupos, parece estar en el modo en que los niños que operan en el nivel de la generalización concreta eran capaces de reorientar su pensamiento para concentrarse en una parte distinta de la operación a través de una secuencia de calusuras.

\section{Estadio de las operaciones formales}

Merece la pena destacar aquí que los alumnos que parecían -ser razonadores formales en el grupo, solían comenzar en cualquiera de los dos lados de la ecuación indistintamente (mientras que los alumnos en el nivel concreto abordaban casi invariablemente en primer lugar el término de la izquierda) y eliminabạn sistemáticamente algunas posibilidades con una primera operación. Un ejemplo típico en un razonador formal de abordar un item como éste

$$
\text { (4"2) } 03=2, *=, o=
$$

era la siguiente: 
algo $03=2$-dedución: o no es + ni $\times$ puesto que forzosamente el resultado debería ser demasiado alto.

algo-3=2 -deducción: 4 * 2 debe ser igual a 5 , lo cual forzosamente no es posible.

o $3=\div 3$

luego si algo

$\div 3=2 \quad$-deducción: 4 * 2 debe ser igual a 6 .

$$
\therefore *=+
$$

esto es $*=+$ y $\mathrm{O}=\div$

Los razonadores formales abordaban el problema muy sistemáticamente, fiable y eficientemente. Parecían aplicar ideas combinatorias al tiempo de tomar conciencia del sistema en su totalidad tal como estaba expresado en el ecuación, más que realizar clausuras específicas y búsqueda de modelos.

\section{SUSTITUCION PRONUMERAL (Collis, 1975b)}

Se descubrió en este estudio que la capacidad de los alumnos para trabajar con pronumerales dependian en gran parte de lo que ellos eran capaces de considerar como "real». Hemos seleccionado el siguiente item como aquel que nos proporciona una imagen más clara para perfilar las respuestas en cada nivel de desarrollo.

Debes decidir si las afirmaciones siguientes son verdaderas siempre, algunas veces o nunca. Haz un círculo alrededor de la respuesta co. rrecta. Si haces un círculo alrededor de (algunas veces) explica en qué casos es cierta la afirmación. Todas las letras representan números enteros o al cero (por ejemplo, $0,1,2,3$, etc.).

1. $\mathrm{a}+\mathrm{b}=\mathrm{b}+\mathrm{a}$ Siempre

Nunca

Algunas veces, esto es, cuando...

2. $\mathrm{m}+\mathrm{n}+\mathrm{q}=\mathrm{m}+\mathrm{p}+\mathrm{q} \quad$ Siempre

Nunca

Algunas veces, esto es, cuando...

3. $a+2 b+2 c=a+2 b+4 c \quad$ Siempre

Nunca

algunas veces, esto es, cuando...

Estadio temprano de las operaciones concretas

En este nivel los alumnos tendían a considerar cada letra como representante de un número y sólo de uno. Su manera de abordar el problema consistía en situar directamente al pronume- 
ral en un número específico: si este único intento no lograba un resultado satisfactorio abandonaban en ese item el trabajo. Por ejemplo, en respuesta al item 1 respondería habitualmente «siempre» sobre la base de ensayar un número para «a" y otro para «b». Al hecho de que el alumno operando en este nivel diera la respuesta requerida "siempre igual" no debe atribuírsele otro significado que "es cierto porque intenté « $\mathrm{a}=3$ » $\mathrm{y}$ «b $\mathrm{b}=4$ " y funcionó». Se comprobó que los dos items siguientes fueron de imposible resolución para los alumnos que utilizaban la estrategia de sustituir cada letra por un número.

\section{Nivel medio de las operaciones concretas}

Los alumnos que operaban en este nivel intentaban un par de números y si satisfacían así la relación sacaban su conclusión sobre esta base. Estos alumnos podían resolver el item 1 porque confiaban en que cierta cantidad de números específicos reemplazaran a los pronumerales: pero eran incapaces de manejar los items 2 y 3.

\section{Estadio de la generalización concreta}

En este nivel los alumnos parecian haber extraído un concepto de número "generalizado" por el que por ejemplo un símbolo "b" podía ser considerado como una entidad por su propio derecho, pero con las mismas propiedades que cualquier número con el que tuvieran experiencia previa. No habian desarrollado aún lo bastante el concepto de pronumeral como para considerarlo como una variable, sino que en lugar de ello pensaban en las letras de los items como representantes de «todos los números en los que uno quiera pensar».

Aún cuando poseían el concepto de número generalizado, los alumnos que operaban en este nivel eran incapaces de afrontar adecuadamente el problema de llevar a cabo la deducción necesaria en el paso final de los items 2 y 3 . Al razonar en voz alta en el item 2, el alumno típico de este nivel parecía destacar "m» $\mathrm{y}$ "q" en cada uno de ambos lados con un comentario como "no importa qué número sean, serán iguales y se eliminan uno al otro». Luego, cuando consideraba $n=p$ su razonamiento seguía esta línea: "Ah, $n$ es igual que p. ¿Cómo puede ser? Hummm, no importa que n y $p$ sean iguales...” y decidía entonces que $n \neq p$, y seleccionaba la respuesta «nunca». Parecía que la respueta correcta no sólo exigía una reconsideración de la información dada que era incapaz de realizar, sino también la introducción de un constructo superordinario que sería preciso para hacer el sistema totalmente autoconsistente. Para él, puesto que "n" variaba a lo ancho de una gran amplitud simultáneamente a la variación de «p» en la misma am- 
plitud, la probabiliad de que ambas se encontraran en un elemento determinado era tan remota como para resultar inconcebible.

También en el item 3 los alumnos que operaban en el nivel de la generalización concreta eran incapaces de realizar la deducción final a partir de la afirmación $2 \mathrm{c}=4 \mathrm{c}$. No eran capaces de concebir un caso en que $2 \times$ (un número) fuera igual a $4 \times$ (un número). Será en el nivel siguiente de abstracción, aquel en que se considere a un pronumeral como una variable, en el que se alcanza la comprensión del cero de modo que el sistema numérico se hace consistente consigo mismo.

\section{Estadio de las operaciones formales}

En el nivel operacional formal (donde el alumno puede cotemplar un pronumeral como una variable) el alumno llega a ser capaz de realizar la deducción final necesaria en los items 2 y 3 . En el item 2 un alumnó capaz de trabajar con variables es capaz de concebir la posibilidad de que, si " $n$ " $y$ " $p$ » varían en una amplitud de números, puede darse una situación en que se mantengan la igualdad y de esta manera el alumno podrá contestar "algunas veces, esto es, siempre que $n=p$ ». Asimismo, en el item 3, el alumno que opere en el nivel formal puede resolver $2 c=4 c$ suponiendo que $\mathrm{c}=0$.

\section{LA OPERACION INVERSA (Collis, 1975a)}

Podemos servirnos del problema de resolver una ecuación simple como $x+5=7$ para ilustrar el concepto que tiene el alumno de la inversa en los diversos niveles de desarrollo.

\section{Estadio temprano de las operaciones concretas}

En el estadio temprano de las operaciones concretas se contempla el problema como una tarea de contar. Para hallar $\times$ el alumno cuenta desde 5 hasta llegar a 7, y registra el número de unidades empleadas. No posee el concepto de la operación inversa. En este nivel, la única noción de la inversa es física, por ejemplo, lo que se ha dejado se puede volver a coger. No se comprenden las implicaciones matemáticas de la operación, de la adición: el signo + es sólo un estímulo para que el alumno se ponga a contar hasta que encuentre la solución.

\section{Estadio medio de las operaciones concretas}

El alumno es este estadio muestra que ve ambas partes de la ecuación como representación de un único número. La operación 
inversa se contempla como un proceso "destructivo». Su razonamiento sigue la linea siguiente: « $\mathrm{x}$ » es un número desconocido pero único, como asimismo « $x+5 »$; el último se ha obtenido "sumando 5» a "X», de tal modo que " $\mathrm{X}$ " puede hallarse sustrayendo "5» de " $\mathrm{x}+5$ " que casualmente es igual a $« 7 »$, y por tanto $\mathrm{x}$ debe ser 2. El alumno contempla el mecanismo de negación como destructivo de la operación original y así el concepto que tiene el alumno situado en el nivel medio de las operaciones concretas, de la inversa, posee una cualidad irreversible.

\section{El estadio de la generalización concreta}

La inversa se contempla en este estadio como un proceso de «deshacer». Es difícil distinguir en la práctica este nivel de pensamiento del que se tiene en el nivel medio de las operaciones concretas,puesto que el alumno ve aún ambas partes de la ecuación como representando un número único, y opera como anteriormente. Sin embargo, el mecanismo de negación se ve como un proceso de "deshacer", esto es, posee una cualidad reversible. Esto permite al alumno que opera en el nivel de la generalización concreta el utilizar la inversión de pasos como método para comprobar su trabajo.

\section{El estadio de las operaciones formales}

En el estadio de las operaciones formales el alumno se concentra en la operación implicada y no necesita contemplar como uníca y empíricamente constante a ninguna de las partes de la ecuación. En el ejemplo que estamos considèrando, puede reemplazarse a 7 por cualquiera de un cierto número de expresiones $(3+4 ; 15-8$; etc.): x podría ser variable o constante. El problema con que tropieza el alumno situado en las operaciones formales es el de hallar la operación que subyace en la operación dada, de tal modo que pueda negarla sin desordenar las relaciones existentes y permitiendo a la vez la posibilidad de volver a la afirmación inicial. En este ejemplo, la sustración es la operación adecuada de anulación y es conveniente utilizar las sustracción de «5" para poder aislar "X»; de una manera similar puede trabajar con el « 7 ", reemplazándolo por comodidad por $2+5$. Podría registrarse su pensamiento así:

$$
\begin{array}{ll}
x+5=7 & \\
x+5-5=7-5 & \begin{array}{l}
\text { (negación de la operación de adición, elección de } \\
\text { un número adecuado y mantenimiento de la rela. } \\
\text { ción). }
\end{array} \\
x+(5-5)=2+(5-5) & \begin{array}{l}
\text { (reemplazamiento de } 7 \text { por una expresión } \\
\text { conveniente y re-asociación). }
\end{array}
\end{array}
$$


$y+0=2+0 \quad$ axioma inverso.

$y=2 \quad$ axioma de identidad

Este ejemplo ilustra la capacidad limitada para manejar las matemáticas del alumno del nivel de las operaciones concretas, si lo comparamos con el alumno que opera en el nivel formal. El concepto que tiene el primero de estos alumnos y el uso que hace de la operación inversa es esencialmente un proceso de negación que opera en una parte del sistema, mientras que el alumno que opera en el nivel formal contempla a la operación inversa como una estrategia recíproca que tiene en cuenta a la totalidad del sistema.

Se han distinguido con claridad (Inhelder y Piaget, 1958) estos dos conceptos de la inversa, "negación» y «reciprocidad», respeto a materiales distintos de los matemáticos. El término de negación se utiliza cuando se anula directamente la operación, y el último cuando se deja la operación sin tocar, aún cuando se neutralice su efecto. Por ejemplo, en el experimento de equilibrado de una barra, el añadir un peso al lado de la izquierda, se niega al quitarlo otra vez, pero puede lograrse el mismo efecto añadiendo el mismo peso al lado de la derecha: una estegia recíproca. El caso es que el alumno no dispone de ambas estrategias hasta que no alcanza el nivel de razonamiento de las operaciones formales. Sugerimos aquí que el método descrito para aislar " $x$ " en " $x+5=7$ ", del que dispone el sujeto que razona en operaciones formales, representa la estrategia recíproca y que los sujetos que piensan con operaciones concretas, utilizan la estrategia de la negación. Aún más, estos últimos no pueden elegir: éste parece ser el nivel más alto al que son capaces de operar. Esta limitación es especialmente significativa ẹn el aprendizaje de las matemáticas.

\section{CLAUSURA (Collis, 1975a)}

Puede rastrearse el desarrollo del pensamiento en el niño en términos de la necesidad que éste tiene por cerrar las expresiones matemáticas. Como ya hemos dicho mućhas veces, en el nivel más temprano, para que una expresión sea significativa para el niño, debe ser susceptible de concluir en un único número. Parece pues que su pensamiento sigue su desarrollo desde un estadio en que debe existir una garantía de clausura (aun cuando no sea necesario realizar una auténtica sustitución) hasta el estadio final, en que se ve a la clausura simplemente como una propiedad matemática y donde el alumno puede operar con variables en las relaciones matemáticas.

En el contexto de este artículo, el nivel de clausura en el que el niño es capaz de trabajar con operaciones depende de su capacidad para considerar el resultado de una operación ( o de una serie 
de operaciones) como único y real. El desarrollo de niveles superiores de razonamiento en matemáticas parece estar estrechamente relacionado con la tolerancia del niño respecto a las operaciones sin concluir. Cuanto más próximo esté el niño al razonamiento concreto temprano, tanto más depende de una clausura inmediata de la operación para poder hacer la situación significativa para sí mismo. Por otra parte, cuanto más cerca esté del nivel más alto del razonamiento adolescente, más patente se hace que alumno puede abstenerse de concluir mientras considera el efecto de las variables en el problema.

Más abajo esbozamos los estadios en el desarrollo de la aceptacion por parte del niño de la falta de clausura respecto a las operaciones matemáticas. La siguiente tabla proporciona ejemplos que ilustran la progresión en el grado de clausura que precisa el alumno a medida que avanza desde el pensamiento concreto hasta el pensamiento operacional formal, en este aspecto de razonamiento matemático.

Dimensión de clausura

Clausura posible y una estrategia eficaz.

Clausura posible pero una estrategia ine ficaz.

La experiencia con los números y las operaciones aritméticas, garantizan la unicidad de los resultados.

Clausura imposible. La unicidad no la garantiza la experiencia.

\section{Ejemplos}

(Se pide al niño que decida en cada caso si las expresiones son equivalentes)

\section{$3+5$ y $2+6$} $5 \times 3$ y $4 \times 4$

$(475+236)$ y $(477+234)$ $(479 \times 231)$ y $(456 \times 231)$

$$
\begin{aligned}
& (a+b) \text { y }(a-1)+(b-1) \\
& (a+1)(b-1) \text { y }(a-1)(b+1) \\
& a \times b \times c \text { y } d \times e \times f
\end{aligned}
$$

\section{Estadio temprano de las operaciones concretas}

Es tal el nivel de clausura que requiere el niño que opera en este nivel, que dos ejemplos conectados por una operación deben ser reemplazados por un tercer elemento al que se reconoce como perteneciente al mismo conjunto, por ejemplo, $3+5$ está próximo a 8. Hablando en términos de números esto significa que en este nivel, las cuatro operaciones de la aritmética elemental son significativas cuando se las utiliza por separado con números pequeños dentro de la experiencia del niño. Sin embargo, el niño ha de po- 
der relacionar, tanto los números como las operaciones, con su mundo físico familiar. El niño de este nivel podría manejar items como los que figuran en la primera fila de la tabla anterior, pero no los siguientes.

\section{Estadio medio de las operaciones concretas}

El nivel de clausura alcanzado en este estadio implica la capacidad de contemplar el resultado de la realización de una operación como necesariamente único, esto es, se reemplazan los dos elementos conectados por una operación por un tercero del mismo conjunto, pero no es necesario realizar factualmente la sustitución para garantizar esto. El alumno puede utilizar ahora números más allá de su alcance empíricamente verificable (por ejemplo $273+472$ ). Puede utilizar también expresiones que impliquen, por ejemplo, dos operaciones que se puedan cerrar secuencialmente, por ejemplo, $(6+4+5)$. En este estadio el sujeto puede dar respuestas correctas a items en las dos primeras líneas de la tabla anterior: puede realizar a menudo items como los de la primera línea sin recurrir a la clausura.

\section{Estadio de la generalización concreta}

En este estadio el alumno sólo requiere que quede garantizada de alguna manera la unicidad del resultado. Por ejemplo, puesto que su experiencia con los números y las operaciones aritméticas le da la seguridad de que puede realizar una clausura en cualquier momento del proceso, puede determinar si el siguiente par de expresiones

$$
\left(\frac{27.9 \times 412}{279}\right) \text { y }\left(\frac{376 \times 412}{376}\right)
$$

son equivalentes, sin clausura. Como decíamos anteriormente, es capaz en este estadio de trabajar con fórmulas como $\mathrm{V}=\mathrm{L} \times \mathrm{B} \times \mathrm{H}$, siempre que se le capacite para tener en cuenta que cada letra representa a un único número y que cada operación binaria puede cerrarse en cualquier punto. Los niños en este estadio ven que se les escapan aún items como los de las última fila de la tabla anterior, pero pueden responder correctamente a los otros ejemplos sin necesidad de clausurar.

\section{Estadio de las operaciones formales}

En el nivel de las operaciones formales se considera a la clausura como una propiedad matemática que puede o no puede exis- 
tir en un conjunto dado. El alumno no relaciona necesariamente a la clausura con su propia realidad empírica, sino que puede aplicarla a elementos abstractos y a operaciones definidas. En este nivel el adolescente puede considerar a la clausura en un sentido formal porque es capaz de trabajar sobre las propias operaciones y no necesita relacionar ni los elementos ni las operaciones con una realidad física. En este nivel el alumno puede enfrentarse con las variables en cuanto tales porque puede evitar el sacar la conclusión final hasta que haya considerado diversas posibilidades: estrategia esencial para obtener una relación distinta de lo que es obtener un resultado único. Este grupo de alumnos puede, obviamente, enfrentarse a todos los tipos de items representados en la tabla anterior.

\section{SISTEMAS MATEMATICOS (Collis, 1975a)}

\section{Sistemas simples y complejos}

Lunzer (1973) distingue entre sistemas simples y complejos. Los sistemas simples son aquellos en que cualquier solución exigida puede ser hallada fijándose en un conjunto de co-variaciones. Los sistemas complejos son aquellos en que están implicados más de un sistema de co-variación, y cualquier solución significativa de un problema planteado depende de la interación de los dos (o más) sistemas. Por ejemplo, los problemas que implican la utilización de la fórmula del área del triángulo pueden ser formulados a cualquiera de los niveles. El niño que opera en el último estadio de las operaciones concretas (generalización concreta) puede trabajar efectivamente con la fórmula $\mathrm{A}=1 / 2 \mathrm{H} \times \mathrm{B}$, a un cierto nivel. Es capaz de reconocer que, dado cualquier triángulo con unidades específicas-que midan "H» $\mathrm{y}$ "B", puede hallar «A». Existe claramente un gran número de posibles triángulos $\mathrm{y}$, consecuentemente, de "A»s, pero es capaz de trabajar con este concepto porque para él es, esencialmente, un solo sistema de co-variación, es decir, que el área cambia según cambia el triángulo, o que " $1 / 2 \mathrm{H} \times \mathrm{B}$ " cambia a medida que cambia el triángulo. Lo que no puede hacer es relacionar los cambios en una o más de las variables " $A$ », «H» $\mathrm{y}$ "B" con los cambios en una o más de las otras. Por ejemplo, no podría resolver porblemas del tipo "A" permanecerá constante $\mathrm{y}$ « $\mathrm{B}$ " cambiará de alguna manera (duplicándose, etc.), cqué ocurrirá entonces con la altura «H»? Este tipo de problema hace que el niño trabaje con la interacción entre dos sistemas. Se varía "B" y debe variarse " $\mathrm{H}$ " en compensación para mantener el producto " $1 / 2 \mathrm{H} \times \mathrm{B}$ " constante. Este tipo de problema,que representa un sistema complejo, no podrá manejarse hasta no alcanzar el nivel operacional formal.

Parece, a la luz tanto de las evidencias experimentales como de la experiencia en el aula, que el nivel de tolerancia de la falta de 
clausura de que dispone el sujeto, determina en gran parte la complejidad del sistema dentro del cual puede trabajar el alumno significativamente. Hasta que no se haya alcanzado el nivel formal, no se considerará a la clausura como una simple propiedad matemática. Esto garantizará que el alumno pueda trabajar significativamente sin clausurar las operaciones y tendrá así la capacidad de manejar sistemas de interacción múltiple en caso necesario, no para obtener un resultado único, sino para considerar las diversas posibilidades que se siguen de la interacción de dos o más sistemas simples, antes de sacar una conclusión final.

Un buen ejemplo de las diferencias en los niveles de pensamiento demostradas por los alumnos que operan en los niveles concretos más avanzados y en los formales, nos lo proporciona la siguiente secuencia de ejercicios. El alumno en el nivel operacional formal puede utilizar su capacidad para reconocer y manejar sistemas de interacción múltiple, para reducir de una manera considerable su trabajo.

«Realiza la siguiente serie de ejercicios en el orden en que se exponen y conservado el trabajo de cada uno de ellos a medida que los vayas completando:

Dado que $V=\pi \mathrm{r}^{2} \mathrm{~h}$,

(1) Hallar V, dado que $\pi=\frac{22}{7}, \mathrm{r}=2, \mathrm{~h}=7$

(2) Hallar $V$, dado que $\pi=$ lo mismo que en el caso (1), $r=$ lo mismo que en el caso (1).

(3) Hallar $\mathrm{V}$, dado que $\pi=$ lo mismo que en el caso (1), r $=$ la mitad de lo que es en el caso (1), $\mathrm{h}=$ lo mismo que en caso (1).

(4) Hallar $h$, dado que $V=$ lo mismo que en el caso (1), $\pi=$ lo mismo que en (1), $r=$ la mitad de lo que es en (1)

En general, en el último estadio concreto (generalización concreta) los alumnos tratan cada ejercicio de manera totalmente independiente. Extraen las nuevas dimensiones y las sustituyen en la fórmula sin darse cuenta del significado de su primer resultado en relación con el cambio en una dimensión de la fórmula. Si se les urge después del segundo ejercicio, algunos convendrán en que se podría haber esperado que la segunda respuesta fuera el doble de la primera, pero la mayoría de ellos continuarán aún realizando sustituciones y cálculos "para asegurarse». En este nivel el niño, como hemos ya indicado varias veces antes, parece ser incapaz de relacionar los cambios en una o más de las variables de una fórmula, con los cambios en una o más de las otras fórmulas. Hasta que no alcanza el estadio del razonamiento formal, el alumno no se da cuenta, y entonces lo hace de inmediato, de la utilidad de su primer cálculo para determinar la segunda respuesta. Con frecuencia su respuesta inmediata será ahora "será el doble que en la primera pregunta", y dará la razón correcta de ella. 


\section{El trabajo dentro de un sistema matemático definido}

El siguiente ejemplo se refiere al caso general en las matemáticas en que se define al sistema en términos de sus elementos, operaciones, reglas, etc. y se espera que el estudiante trabaje dentro de este sistema sin referencia a ninguna realidad fuera del propio sistema.

Consideremos la siguiente cuestión: «a, b, c, ..., etc. puede ser cualquiera de los números $0,1,2,3, \ldots$ etc. $\mathrm{y}$ * es una operación del tipo $a{ }^{*} b=a-2 \times b$. Examina cada una de las siguientes afirmaciones e indica cuando es cierta cada una de estas afirmaciones.

(1) $a$ " $b=b$ " $a$

(2) $a *(b * c)=(a * b) * c$

(3) $a * d=a$

(4) $a^{*}(b+c)=\left(a^{*} b\right) * c$

(5) $\mathrm{a}+(\mathrm{b} * \mathrm{c})=(\mathrm{b} * \mathrm{c})+\mathrm{a}$

Parece razonable sugerir que sería preciso que un alumno estuviera operando al nivel formal para trabajar dentro de un sistema matemático definido como el expuesto arriba. En un estudio (Collis, 1975 a) realizado para comprobar esta hipótesis, se halló que sólo el grupo de edad de 16-17 años, al que se presentó como perteneciente al estadio de las operaciones formales sobre criterios arreglados para firmar tal cosa, parecía tener algún grado de éxito con estos sistemas. Los resultados del estudio revelaron también que los alumnos que operaban por debajo del nivel formal tendían a ignorar el sistema definido dado y razonaban por analogía con un sistema familiar. Más abajo damos ejemplos de sus respuestas.

\section{Estadios temprano y medio de las operaciones concretas}

Los alumnos que operan a estos niveles tienden a ignorar la operación definida y sustituyen las operaciones binarias de aritmética elemental. En el nivel concreto temprano, una de las respuestas típicas era "no sé", pero en general, los alumnos que operaban en los niveles temprano o medio ( 9 años de edad y mayores) sustituían el sistema dado por otro familiar. Por ejemplo, una respuesta típica a los items 1 y 2 era «Es verdaderà, cuando * significa $+o \times$. Parecía que un alumno razonador concreto no podía aceptar el sistema como definido y trabaja dentro de las limitaciones impuestas, sino que en lugar de ello precisaba remitirse a la experiencia concreta previa con materiales de un tipo superficialmente similar.

\section{Estadio de la generalización concreta}

Los alumnos que operan en el nivel de la generalización concreta mostraban que eran conscientes del hecho de que era preci- 
so usar la operación definida como tal y no trasladarla a una operación más familiar, pero no tenían suficiente control del sistema como para ser capaces de deducir los resultados correctos. items:

Estos son ejemplos de respuestas típicas a los dos,primero

(1) Cuando $b * a=a-2 \times b$

o

Cuando $a+2 \times b=b+2 \times a$

(2) Cuando $a^{*} \mathrm{~b}=\mathrm{b} * \mathrm{c}$

0

Cuando $\mathrm{a}+2(\mathrm{~b}+2 \times \mathrm{c})=(\mathrm{a}+2 \times \mathrm{b}) 2 \times \mathrm{c}$

Parece como sí, en este estadio transitorio, el alumno pudiera generalizar suficientemente a partir de su experiencia con operaciones como para utilizar la operación definida correctamente, pero era incapaz de ir más allá de la información que se le presentaba para realizar las deducciones necesarias sobre las variables.

\section{Estadio de las operaciones formales}

Aun cuando estos estudiantes eran capaces de trabajar correctamente dentro del sistema definido, también en este nivel algunos items se alcanzaban mucho más rapidamente que otros. Los items 1,3 y 5 se alcanzaban antes que los items 2 y 4 . Este resultado es consistente con la postura de muchos investigadores de que los indivíduos necesitan un alto nivel de razonamiento abstracto para realizar operaciones sobre operaciones. Los items 1,3 y 5 requieren que el alumno trabaje con una sola operación definida, pero los items 2 y 4 exigen que el alumno trabaje con dos operaciones definidas al menos en la expresión matemática objeto de investigación.

\section{RESUMEN}

El modelo piagetiano de los estadios del desarrollo cognitivo ha proporcionado un importante estímulo a los psicólogos que trabajạn en el área del conocimiento. Sin embargo, los problemas le han perseguido. El teórico o investigador se encuentra con inconsistencias o lagunas inexplicadas cuando intenta replicar el trabajo original sobre el que se ha fundamentado la teoría. El experto educativo interesado en aplicar la teoría al curriculum práctico y a las situaciones de empleo de los métodos educativos, se encuentra con que las extrapolaciones que debe realizar son inadecuadas. Este artículo intenta realizar un asảlto preliminar a 
ambos problemas. Presenta, en primer lugar, una formulación de los principios básicos en términos educativos de las matemáticas escolares que debería ser de alguna ayuda ante el último de estos problemas. En segundo lugar, y al tiempo que realiza esa tarea, permite al teórico contemplar el problema desde un ángulo ligeramente distinto.

La primera parte de este artículo sostiene el punto de vista de que hay que considerar a las matemáticas escolares, ya incluso desde su más temprana introducción en la clase, como un sistema lógico, como una estructura jerárquica de relaciones. A la hora de planear método y programas es preciso tener esto en cuenta, al igual que el estadio del desarrollo cognitivo del niño. Tomados juntos, estos dos aspectos apuntan a la necesidad de que, el sistema lógico que constituyen las matemáticas, tenga un fundamento concreto en el sentido piagetiano del término, y a todos los niveles dentro del estadio concreto. Pero decir esto es no decir nada si no llegamos a definir las limitaciones en el funcionamiento lógico del niño en los diversos niveles. De una manera general esta tarea se aborda en la segunda parte del artículo.

La segunda parte comienza ocupándose más específicamente de las matemáticas escolares en relación con los estadios de desarrollo y se sugiere que el modelo de procesamiento de la información proporciona una vía fructifera de contemplar el problema de la explicaciónde los fenómenos de los estadios. Subrayamos aqui nuestro interés en analizar la respuesta de un sujeto a un problema y de alinearla con un nivel específico de funcionamiento. El nivel de funcionamiento indicado tiene por arriba un límite inferior. En otras palabras, una persona que está clasificada como un razonador formal puede dar en general, en una situación determinada, una respuesta típica de un nivel mucho más bajo. Las razones para este fenómeno pueden variar desde el humor de la persona en èse momento hasta la falta de familiaridad con las limitaciones de la tarea.

El artículo se cierra relacionando un cierto número de importantes conceptos matemáticos para el desarrollo y la comprensión matemáticas de un sujeto, con los diversos estadios piagetianos. Parece claro que cada movimiento a lo largo del continuo desde el razonamiento preoperacional hasta el operacional-formal, en el caso de todos los conceptos considerados, implica el ser capaces de afrontar tanto conceptos cada vez más abstractos en lo relativo a los elementos, como una creciente complejidad en las operaciones con esos elementos. Ambas cosas están inexplicablemente unidas con los estadios de desarrollo. 


\section{Referencias}

CASE, R. (1977). Intellectual development from birth to adulthood: a neo-Piagetian interpretation, ar ticulo presentado en el Thirteenth Annual Carnegie Symposium on Cognition.

Collus, K. F. (1974). Cognitive Development and Mathematics Learning. The Psychology of Mathematics Education Series: Chelsea College, University of London.

Collis, K. F. (1975 a). A Study of Concrete and Formal Operations in School Mathematics: A Piagetian Viewpoint. Melbourne: A.C.E.R. Research Series N.॰ 95.

Collis, K. F. (1975 b). The Development of Formal Reasoning. Newcastle: University of Newcastle.

Collis, K. F. and BigGs, J. B. (1978). Classroom Examples of Cognitive Development Phenomena - Grade 3 through Grade 12. Informe de Investigación en redacción informal.

Hallam, R. N. (1967). Logical thinking in history, Educational Review. Vol 19, N.०3, pp. 183-202.

HALLAM, R. N. (1969). Piaget and moral judgments in history, Educational Research, Vol 11, N.³, pp. 200.206.

INHELDER, B. y PIAGET, J. (1958). The Growth of Logical Thinking from Childhood to Adolescence. Londres: Rutledge \&c Kegan Paul.

JURD, M. E. (1970). Structures of adolescent thought with history-type material. Tesis inédita, New. castle: Universidad de Newcastle.

LUNZER, E. A. (1973). The development of formal reasoning: some recent experiments and their implications. En: Frey, K. y Lang, M. (Eds) Cognitive Processes and Science Instruction. Berna Huber \& Baltimore.

MASON, J. S. (1974). Adolescent judgments as evidenced in response to poetry, Educational Review, Vol 26, N. 2 , pp. 124.39.

PEeL, E. A. (1960). The Pupil's Thinking. Londres: Oldbourne.

PEEL, E. A. (1975). Predilection for generalizing and abstracting, British Journal of Educational Psychology, $45,177.88$.

RHYs, W. T. (1966). The development of logical thought in the adolescent with reference to the teaching of geography in the secondary school, tesis inédita, Universidad of Birmingham.

SUTTON, R. S. (1962). Behaviour in the attainment of economic concepts I, Journal of Psychology, 56, 37.46.

SUTTON, R. S. (1964). Behaviour in the attainment of economic concepts II, Journal of Psychology, 58, 407.12.

WASON, P. C. (1974). The theory of formal óperations - a critique. Comunicación presentada en la London School of Economics. 